\title{
Affronter la difficulté de notre relation à l'animal : esquive, imagination et projection
}

Dealing with the Difficulty of our Relations to Animals: Deflection, Imagination and Projection

\section{Alexis Anne-Braun}

\section{(2) OpenEdition \\ Journals}

Édition électronique

URL : https://journals.openedition.org/cps/4634

DOI : $10.4000 /$ cps.4634

ISSN : 2648-6334

Éditeur

Presses universitaires de Strasbourg

Édition imprimée

Date de publication : 30 mai 2021

Pagination : 61-83

ISBN : 979-10-344-0088-1

ISSN : $1254-5740$

\section{Référence électronique}

Alexis Anne-Braun, «Affronter la difficulté de notre relation à l'animal : esquive, imagination et

projection », Les Cahiers philosophiques de Strasbourg [En ligne], 49 | 2021, mis en ligne le 30 mai 2021 consulté le 08 novembre 2022. URL : http://journals.openedition.org/cps/4634 ; DOl : https://doi.org/ $10.4000 /$ cps.4634

\section{(c) (i) (ㅇ)}

Creative Commons - Attribution - Pas d'Utilisation Commerciale - Partage dans les Mêmes Conditions 4.0 International - CC BY-NC-SA 4.0

https://creativecommons.org/licenses/by-nc-sa/4.0/ 


\section{Affronter la difficulté de notre relation à l'animal : esquive, imagination et projection}

Alexis Anne-Braun

Être vu, nu, par un chat; essayer de savoir quel effet cela fait d'être une chauve-souris; penser les modes de cohabitation et de compagnonnage que nous formons spontanément avec les animaux, en dépit de toutes les violences que nous leur faisons subir, constituent autant d'expériences de pensée limites qui ont ponctué le discours philosophique sur l'animalité au $\mathrm{Xx}^{\mathrm{e}}$ siècle, autant d'expériences qui semblent traduire une égale difficulté à raccrocher la pensée à la réalité de l'animal, comme si cette dernière, dans la complexité des différences et des contradictions qu'elle contient, lui opposait une forme de résistance susceptible de nous plonger dans le vertige, le scepticisme ou la schizophrénie morale. Avec le caillou biffé du lézard de Heidegger (qui est sur le caillou sans avoir de point de vue sur le caillou), la chatte de Derrida ou la chauve-souris de Nagel, il en va ainsi peut-être du même vertige de la pensée - ce vertige et cette "Unheimlichkeit», dit Heidegger, qui est en fait "celui du questionnement sur l'animal $»^{1}$.

Il n'est pas étonnant que Cora Diamond fasse un cas justement - le cas de l'animal - de ce qu'elle nomme "une difficulté de la réalité", c'est-à-dire un point d'achoppement entre la pensée et ce qu'elle essaye de saisir, le réel, qui est susceptible de nous faire perdre nos concepts. Pour la philosophe américaine, la difficulté de la réalité en question, qui a ses versions morales comme ses versions épistémologiques, concerne le fait que l'animal puisse nous être à la fois si semblable et si dissemblable; la stupeur devant le fait que notre pensée ne parvienne pas à saisir ce que cela veut justement dire qu'il existe de tels êtres et quelles en sont

1 Jacques Derrida, L'Animal que donc je suis, p. 212.

Les Cahiers Philosophiques de Strasbourg, i / 202 I 
les conséquences, morales et épistémologiques. Je me propose, dans ce texte, d'explorer les voies de cette difficulté, qui sont souvent des voies contournées ou des déviations - comme le fait remarquer Cora Diamond. L'idée est aussi de réfléchir aux moyens dont la philosophie dispose pour affronter cette difficulté, sans dénégation et sans déviation, car cette difficulté de la réalité pourrait tout aussi bien être une difficulté de la philosophie. De fait, la philosophie n'est jamais aussi urgente que là où elle est en question, là où les questions qu'elle formule s'éprouvent comme un vertige, là où elle affronte un non-sens, fait l'expérience d'une perte de concepts. De ce point de vue, la question animale est aussi une question adressée à la philosophie et à ses méthodes.

En suivant la piste ouverte par Cora Diamond, j'examinerai deux difficultés soulevées par une certaine façon de recourir aux arguments philosophiques. La première consiste à ignorer des différences qui ont leur importance morale, la seconde consiste à nier certaines ressemblances sur fond de scepticisme. Ces deux attitudes ont en commun d'esquiver la difficulté, après l'avoir entrevue. Il se trouve qu'elles témoignent moins d'un mauvais usage de l'argumentation que de son usure.

\section{Esquives et différences morales}

Une partie de la réflexion de Diamond s'inscrit dans une discussion éthique au sujet de ce que signifie le fait de "manger de la viande $»^{2}$. Par-delà cette dimension morale, il convient de relever que les concepts qui sont en jeu dans ce contexte (au risque pour certains d'être perdus ou de se perdre) sont des concepts d'une portée en fait beaucoup plus générale, des concepts tels qu'être en vie et être mortel, avoir un corps ou se projeter dans le corps de l'autre. Il est remarquable que ces analyses croisent justement certaines idées avancées par l'éthologie contemporaine dans ce qu'elle a de plus inventif.

On ne saurait comprendre la position originale défendue par Cora Diamond sans d'abord déterminer ce contre quoi elle se pose. Dans la lignée de Peter Singer, un certain nombre de philosophes contemporains se sont proposés de répondre clairement à la question formulée au $\mathrm{XvIII}^{\mathrm{e}}$ siècle par le philosophe britannique Jérémy Bentham: «Les

2 Cora Diamond, L'Importance d'être humain, «Manger de la viande, manger des gens", p. 109-132. 
animaux peuvent-ils souffrir?». Sans vouloir minimiser ni le changement radical de perspective ouvert par la question benthamienne ${ }^{3}$, ni le rôle que jouèrent les différents courants utilitaristes (ou des défenseurs des droits) dans la lutte en faveur de la libération animale, Cora Diamond identifie plusieurs difficultés dans la démarche argumentative de Singer - des difficultés qui ont trait à l'acte même d'argumenter.

Lidée, très simple, de Peter Singer consiste à prendre en compte les préférences et intérêts de tous les êtres vivants (sans préjugés spécistes), dans le calcul utilitariste de ce qu'est une action juste. Les théoriciens du droit, bien qu'il existe des désaccords avec la position de Singer sur ce que peut signifier pour un être vivant le fait d'être une personne morale et juridique ${ }^{4}$, procèdent d'une manière identique, en particulier dans leur rejet des préjugés spécistes. Par manière identique ou procédé, j'entends le recours à l'argument des cas marginaux: comparer les capacités (par exemple l'usage d'une parole à propos), intérêts et préférences de certains mammiferes aux intérêts et préférences des fous, des bébés, des hommes diminués5. Le risque, insiste Diamond, c'est que se perde, à ces occasions, un certain sens de l'être humain et de son «importance».

3 Sur ce changement de perspective, voir par exemple le commentaire de Jacques Derrida dans L'Animal que donc je suis: «C'est en pensant à la source et aux fins de cette compassion que quelqu'un comme Bentham, c'est bien connu, a proposé il y a deux siècles de changer la forme même de la question de l'animal, telle qu'elle domine aussi le discours de la tradition, aussi bien dans son argumentaire philosophique le plus raffiné que dans le langage courant du sens commun. [...] La question préalable et décisive serait de savoir si les animaux peuvent souffrir. Can they suffer?» (p. 48). Il est à noter que Cora Diamond pense que le doute devant cette question est «hors sujet": cf. Cora Diamond, L'Importance d'être humain, p. 111.

4 Sur ce point je renvoie à: Peter SINGer, "Libération animale ou droit des animaux?»; et Tom Regan, "Pour les droits des animaux», in: HichamStéphane Afeissa, Jean-Baptiste Jeangene Vilmer (dir.), Philosophie animale: différence, responsabilité et communauté.

5 Cora Diamond, L'Importance d'être humain, p. 110: «En fait Singer et Regan soutiennent tous les deux que si nous retenons, aux fins de justification pour un traitement différentiel, des choses comme l'incapacité des animaux à user de la parole, nous devrions traiter comme des animaux les membres de notre propre espèce qui (disons) souffrent d'une déficience cérébrale propre à empêcher le développement de la parole - devrions permettre leur utilisation comme animaux de laboratoire ou comme nourriture ou comme ce qu'on voudra». 
Or, il s'avère que ce sens de l'être humain, s'il est suffisamment travaillé par l'imagination et détaché de tout essentialisme biologique, permet de situer autrement le problème de nos relations aux animaux. Ainsi, à partir du rejet d'un mode de l'argumentation philosophique, Cora Diamond est amenée à re-problématiser notre relation à l'animal, c'està-dire la manière dont nous le reconnaissons, nous y reconnaissons et pouvons (ou non) le faire accéder à une forme de vie humaine qui serait par-là élargie.

Ainsi, certains philosophes, remarque-t-elle, prennent le parti d'ignorer la différence qu'il y a entre les humains et les animaux en vue de reconnaître abstraitement des droits ou des intérêts à ces derniers. Seulement, faute de marquer cette différence, ils passent à côté de ce qui est véritablement en jeu dans les attitudes que nous avons avec des êtres qui sont pris dans le tissu de relations que nous reconnaissons comme la vie humaine (donner des noms, enterrer les morts, ne pas les manger). Il en va, en fait, de la perception de ce qui «importe dans notre relation aux autres êtres humains ou autres animaux $»^{6}$. De la part des théoriciens de la libération animale, cette ignorance est en partie volontaire, puisqu'elle est l'horizon du combat (qui n'est d'ailleurs pas illégitime) contre certains préjugés spécistes. Seulement, ne pas reconnaître que notre vie morale est affaire de frontières déposées et inscrites dans le langage, dans nos façons de nous rapporter à la réalité, revient à refuser d'appréhender le problème dans toute sa complexité.

On ne saurait par exemple comprendre certaines versions du végétarisme si on occulte le fait qu'il en va pour les végétariens de tout autre chose que d'un calcul d'intérêt (d'intérêts élargis à tout le vivant). Un utilitariste ne mangerait pas de la viande humaine s'il avait l'assurance que les intérêts de la personne qu'il mange n'ont pas été lésés. Pourquoi ne pas voir que c'est quelque chose d'identique qui se produit dans le végétarisme: un dégoût ou un interdit qui ne sauraient être qualifiés abstraitement (qualifiés comme une mauvaise action par exemple). Pourquoi d'ailleurs ne mangeons-nous pas nos animaux domestiques? Précisément parce que, si nous le faisions, nous n'aurions pas le même concept d'animal domestique. Ce que nous mangeons ne fait pas partie du même concept que ceux avec qui nous vivons, en leur donnant des noms, des ordres, des caresses, de l'attention:

$6 \quad$ Ibid, p. 112. 
«Un animal domestique n'est pas quelque chose qu'on mange, on lui donne un nom, on le laisse entrer chez nous et on peut lui parler d'une façon qui n'est pas normalement en usage avec les vaches ou avec les écureuils. En somme, on lui donne une partie du caractère d'une personne. (Ceci pourrait être plus ou moins sentimental; mais n'a nul besoin d'être sentimental). Traiter les animaux domestiques de cette façon n'est pas du tout l'affaire de reconnaître quelque intérêt qu'auraient les animaux domestiques à être ainsi traités. Il n'y a pas une classe d'êtres, les animaux domestiques, dont la nature, dont les capacités sont telles que nous leur devons de les traiter de ces façons ${ }^{7}$.

À côté donc des ressemblances qui existent entre tous les animaux (et au nombre desquelles il faut compter le fait d'être en vie, d'avoir des préférences, de souffrir et de craindre la mort - encore que bien sûr, à prendre le mot animal dans toute sa généralité, on soit vite amené à reconnaître des ressemblances qui s'amenuisent sur les bords), il existe une autre série plus élémentaire de différences qui apparaissent dans le langage ordinaire. Ce sont ces différences qui rendent visible le tissu ou la texture de notre forme de vie - différences qui apparaissent dans ce que signifie par exemple, pour un être humain, d'avoir un nom et de ne pas être mangé, d'avoir des funérailles ou d'être un camarade; bref, tout ce qui fait le concept "d'être humain» dans son emploi linguistique ordinaire . $^{8}$

Est-ce une autorisation particulière à manger ce qui n'entre pas sous ce concept ou à maltraiter les animaux et à leur dénier tout ce qu'on rassemble ailleurs sous le nom de dignité humaine? Descartes semble le penser, lui dont "la pieuse opinion en faveur des hommes " ${ }^{9}$ œuvre à les délivrer du soupçon de crime dès qu'ils mangent de la viande. De prime abord, la question est légitime, d'autant plus si le rappel de ces différences (ce qu'on mange/ce qu'on ne mange pas, viande/défunt) entre dans ce

$7 \quad$ Ibid, p. 117.

8 Il y a dans les débats métaéthiques des auteurs qui mettent au contraire l'accent sur ces différences, des différences qui deviennent décisives lorsqu'il s'agit de définir quels sont nos devoirs dans des situations morales particulières. Je pense en particulier à certains textes de William David Ross dirigés contre le caractère toujours trop abstrait de l'utilitarisme: $c f$. William David Ross, The Right and The Good, p. 22.

9 Lettre à Henry Morus, 5 février 1649, René Descartes, Correspondance, 2, OC VIII, Gallimart, TEL, 2013, p. 648. 
qui fait la texture de notre vie morale. Pour autant, il n'y a pas de sens à vouloir fixer l'essence et le propre de l'humain comme animal rationnel, animal parlant ou animal qui aurait une quelconque capacité qu'eux n'auraient pas; pas de sens non plus à circonscrire ce concept d'être humain sous la catégorie de l'Homo Sapiens. Il est donc nécessaire de penser à nouveaux frais ces problèmes de ressemblance et de différence, les penser hors des catégories métaphysiques traditionnelles.

Déjà, ces différences n'ont pas à être découvertes, puisqu'elles se donnent d'emblée dans la forme que prennent nos vies et dans le langage sur lequel elles s'adossent. Il y a "eux", et il y a "nous", pourrait-on dire. Seulement derrière "eux" et derrière "nous", il est trompeur de chercher d'abord ou exclusivement des espèces biologiques. "Eux" et "nous", comme le signale notre langage, sont des genres d'être avec lesquels on entre différemment en relation, des genres d'être par exemple que nous mangeons et des genres d'être que nous ne mangeons pas; des genres d'être à qui on reconnaît l'indépendance, l'autonomie et la dignité et des genres d'être à qui l'on dénie ces mêmes choses. Au fond, et en dépit de toutes les similitudes existantes, c'est nous qui formons le concept de ces différences. Il s'avère d'ailleurs que l'humanité elle-même n'est pas toujours contenue entièrement dans ce "nous", que le langage manie avec une confusion parfois coupable. Sinon, comment comprendre que l'on puisse réduire certains hommes en esclavage ou les exterminer? Que fait-on alors si ce n'est dénier à l'homme, dans une situation ou une autre, lorsqu'il est esclave, banni, hors-la-loi ou migrant, ce qui entre normalement sous son concept? Cora Diamond écrit ainsi :

"Certains modes de réaction peuvent être perçus comme des façons de retirer de certains animaux ("vermine"10) ou d'animaux en certaines circonstances (le danger) ce qui autrement appartiendrait au fait de les reconnaître comme des animaux, tout comme la notion de l'ennemi ou de l'esclave peut impliquer le retrait hors de la personne visée d'une partie de ce qui relève de sa reconnaissance comme être humain. [...] Ce qui se produit dans ces cas est que nous avons quelque chose que nous ne traitons pas comme ce qu'il - d'une certaine façon - est $»^{11}$.

10 On pourrait bien sûr continuer cette liste: "nuisible", "bétail", "viande".

11 Cora Diamond, L'Importance d'être humain, p. 128. 
Si donc la texture de ce "nous» est ouverte et irréductible à toute définition biologique ou métaphysique (comme est «labile» l'extension du concept de créature vivante ${ }^{12}$ ), il n'est pas interdit d'essayer de capturer d'autres aspects de la réalité à partir de lui, en particulier des formes de vie qui impliquent le fait d'avoir un nom, d'être un compagnon, de vivre avec nous ou d'avoir des funérailles - ou plus généralement encore, le fait d'avoir un corps et d'être voué à la mort; ce que nous faisons ordinairement du reste, et très spontanément, avec certains animaux ${ }^{13}$. Plutôt donc que d'esquiver la réalité de notre différence avec l'animal au nom d'une définition abstraite de la vie, il serait plus pertinent de considérer tous les problèmes de projection ou de transposition que cette proximité étrange soulève, et d'en parcourir et redessiner souvent les frontières ${ }^{14}$, selon qu'on s'intéresse à certaines ressemblances en particulier, signalées par le langage. C'est ce qu'entreprend aussi de son côté l'éthologie contemporaine sur la base de l'analyse des conduites,

12 Ibid, p. 125. L'idée qu'un concept puisse avoir une texture ouverte ou une extension variable est liée à la conception du langage que se fait Cora Diamond et qui est d'inspiration wittgensteinienne (plus exactement héritée de Stanley Cavell). Il est clair que le concept de projection mis en avant dans la seconde partie de cet article (en rapport avec l'idée d'imagination sympathique ou d'imagination morale) est lié aussi à cette conception du langage, à ce que signifie projeter un mot ou un concept dans de nouveaux contextes.

13 On n'est pas forcé non plus d'étendre ce concept du «nous» dans toutes les directions. Le concept limité "d'être humain" aura sa pertinence dans la plupart des contextes. Pour ne donner que deux exemples empruntés à Diamond: il sera pertinent pour décider avec quel genre d'adulte nous pouvons passer un contrat ou avec quel genre d'adulte nous pouvons avoir une sexualité accomplie.

14 Sur cette question des frontières, je renvoie à ce que Jacques Derrida dit de la limitrophie dans L'Animal que donc je suis: «La limitrophie, voilà donc le sujet. Non seulement parce qu'il s'agira de ce qui pousse et croît à la limite, mais de ce qui nourrit la limite, la génère, l'élève et la complique. Tout ce que je dirai ne consistera surtout pas à effacer la limite, mais à multiplier ses figures, à compliquer, épaissir, délinéariser, plier, diviser la ligne justement en la faisant croître et multiplier" (p. 51). Pour une éthique de la différence avec l'animal, je renvoie également au chapitre sept de l'essai d'E. Bimbenet, Le Complexe des trois singes. Essai sur sur l'animalité humaine (p. 285-333). 
lorsqu'elle recherche le commun dans des rapports parfois déconnectés de l'ancestralité ou de l'histoire évolutive ${ }^{15}$.

Tout l'enjeu, on le comprend, est de parvenir à faire passer le vivant du bon côté de la frontière (du côté de ce "nous" que l'on ne mange pas et qui, comme nous, possède un corps propre et une certaine indépendance). Or, comme je l'ai déjà remarqué, nous avons des façons parfois «confuses» de nous rapporter aux autres. Diriger notre attention sur ces différences qui comptent moralement ne doit pas nous empêcher de voir par exemple l'hypocrisie avec laquelle nous nous rapportons en général aux animaux, produisant artificiellement certains découpages: entre les bêtes que nous mangeons et les animaux que nous aimons lesquels peuvent être d'ailleurs les mêmes, ce qui n'est pas sans manifester ou occasionner une espèce de schizophrénie morale ${ }^{16}$. C'est bien en tout cas sur ces confusions qu'un éclaircissement doit être apporté, sur ces confusions que doit travailler notre imagination morale; car il est clair que «les animaux - ces objets sur lesquels nous agissons - ne sont pas

15 Voir par exemple la manière dont Baptiste Morizot annexe certaines pratiques de l'éthologie contemporaine à son approche diplomatique dans Les Diplomates. La mise en évidence du commun de la vie chez les mammiferes donne lieu à des pages très inspirantes: «Nous partageons en biomorphie, à différentes échelles, avec le monde mammifere (et animal dans une certaine mesure) les grandes étapes initiatiques de l'existence. Naître, être caregiver, jouer et apprendre, la joie de chercher ce qui est bon pour nous, la peur de ce qui est mauvais, l'affection pour les proches, l'aversion pour d'autres, l'indifférence tranquille, la vibrance du désir, quelque chose comme la rencontre amoureuse, se tailler une vie, une niche, un milieu, être parent, s'insérer dans un collectif ou partir, former nos relations politiques avec les autres, faire le deuil des proches, puis décliner, avoir plus de passé que d'avenir, voir les jeunes monter, trouver comment interagir avec eux, mourir» (Baptiste Morizot, Les Diplomates, p. 59).

16 Sur ce point voir aussi l'article de Gary Francione, «Prendre la sensibilité au sérieux", in: Philosophie animale, p. 196: "Nombreux sont ceux qui parmi nous considèrent comme des membres de la famille les êtres nonhumains avec lesquels ils vivent. Et pourtant, nous retournons notre steak dans la poêle pour finir la cuisson, puis plantons gaillardement notre fourchette dans la chair d'autres êtres non-humains, alors même qu'ils ne se distinguent de façon significative sous aucun rapport des animaux que nous aimons». Je renvoie également au travail de Susan Isaacs, Intellectual Growth in Young Children (p. 160-162), que commente Cora Diamond dans L'Importance d'être humain et dans l'essai "L'expérimentation sur les animaux" (in: L'Esprit réaliste, p. 471). 
donnés à notre pensée indépendamment de cette foule de manières de les penser et d'y réagir ${ }^{17}$, foule de manières qui peuvent être amendées et travaillées dans des directions très différentes.

Pour ce faire, il convient de reconnaître dans l'animal, non pas un sujet ayant telle ou telle capacité et donc tant et tant d'intérêts, mais un être qui partage avec nous le fait d'être en vie - d'être un "fils de la vie" pour reprendre l'image du poète Walter De la Mare que commente Diamond dans son essai, un être autonome, sujet possible de verbes d'action (marcher, courir, voler, fuir, fureter, pister, dormir, rêver même et tout le bestiaire des cris $)^{18}$, qui partage aussi avec nous l'inévitabilité de la mort, bref une "créature vivante». C'est moins que le fait de vivre avec nous ou que d'avoir un nom, mais ce commun-là, c'est aussi au titre de ce qui fait l'importance d'être humain qu'il peut être défendu ${ }^{19}$. Comme le remarque Diamond, nous ne pouvons comprendre ce que cela fait d'avoir une vie corporelle qu'à partir de ce que cela signifie d'avoir un corps qui soit justement humain, un corps vulnérable, excitable, exposé à la mort ou à toute autre déception moins dramatique. C'est une autre

\section{Cora Diamond, L'Importance d'être humain, p. 127.}

18 Jean-Christophe Bailly s'est intéressé tout particulièrement aux verbes animaux dans un texte intitulé "Les animaux sont des maitres silencieux", repris depuis dans Le Parti pris des animaux: «Étrangement, à cet univers pour nous fermé et sans noms, les verbes, sous leur forme infinitive semblent pouvoir introduire un peu mieux: génériques, c'est comme s'ils se situaient dans une sorte de plein-emploi du sens, antérieur à la dénomination proprement dite: là où les substantifs ou les adjectifs se démarquent comme des points, les infinitifs adviennent comme des lignes, ou font advenir des lignes, et ces lignes, non seulement on peut dire que les animaux les écrivent, mais aussi qu'on peut les suivre» (Jean-Christophe BaIlly, Le Parti pris des animaux, p. 86).

19 Cora Diamond, L'Importance d'être humain, p. 124: «L'attitude à l'égard des animaux comme compagnons mortels dans la vie sur cette terre [...] dépend d'une conception de la vie humaine. C'est l'extension d'une notion non biologique de la vie humaine». Bien sûr, si un homme ne se rend pas capable de cette extension (parce qu'il a une «imagination morale limitée») et si le concept de "créature semblable» ne lui dit rien, le discours de Cora Diamond sera de peu de portée. Ce qu'elle reconnaît elle-même très bien. Pas plus que l'appel à l'imagination morale, les arguments philosophiques ne parviennent d'ailleurs à convaincre tout le monde. À ce sujet, voir son texte "Rien que des arguments?», in: L'Esprit réaliste, p. 391-415, ainsi que l'introduction au texte de Stephen MulHall, The Wounded Animal, J.M. Coetzee and the Difficulty of Reality in Litterature \& Philosophy. 
manière de dire que nous ne pouvons comprendre l'animal qu'à partir de ce que signifie pour nous le fait d'être un humain. Au contraire, faire peu de cas de cette signification, sur le modèle d'un antihumanisme de combat, nous mettrait dans une situation difficile pour reconnaître le genre d'être que nous avons en commun avec l'animal. Penser la relation à l'animal à partir de notre humanité n'est pas, non plus, une autre façon de mobiliser le concept kantien de «devoir indirect» ou de "dignité humaine», selon une logique qui n'est pas dénuée de cruauté tant elle s'acharne contre le rôle que peut jouer aussi la sensibilité en morale ${ }^{20}$. C'est plutôt que nous-mêmes en tant qu'humains, et parce que nous sommes aussi pour cela des vivants, nous avons à reconnaître la différence qu'il y a entre mener une existence où, comme dans les tableaux de Paulus Potter ${ }^{21}$, nous entretenons des relations riches avec les autres êtres vivants, et mener une existence où ce concept de la vie, ce tissu de relations qui noue ensemble vibrance du désir, crainte, souffrance, joie, vulnérabilité, jeu, est ignoré et esquivé.

Il est dommage que ce soit justement toute cette texture qui soit occultée dans une définition abstraite de la vie et de la capacité à souffrir qui, abolissant toute frontière, ignore aussi toutes les différences. Dommage et significatif car sur la question de notre relation à l'animal, la philosophie s'est souvent esquivée. Et comme on va le voir, il y a plusieurs manières de s'esquiver.

\section{Esquives et projections}

Face à l'animal et face à la difficulté de le penser, en effet, nous nous esquivons. C'est dans un texte consacré à l'auteur sud-africain J.M. Coetzee que la philosophe Cora Diamond est au plus près de cette intuition - une intuition qui est également partagée par Elizabeth Costello, le personnage de romancière que Coetzee met fictivement en

20 Sur Kant et la cruauté de sa morale, voir le commentaire que Jacques Derrida fait du texte d'Adorno, Beethoven. Philosophie de la musique, dans L'Animal que donc je suis, p. 139.

21 Je pense à la richesse de l'imbrication entre l'homme et l'animal dans les tableaux animaliers du peintre hollandais, que convoque Cora Diamond dans son texte «Perdre ses concepts» (L'Importance d'être humain, p. 169). 
scène dans une conférence portant sur la vie des animaux ${ }^{22}$. À juste titre, Diamond présente la romancière Elizabeth Costello comme un «animal blessé», parlant non seulement pour les animaux, à leur place, mais aussi comme l'animal qu'elle est. Il en résulte un déplacement discursif intéressant. En tant qu'animal blessé, l'autre de l'homme à qui, dans une continuité philosophique remarquable mais aussi pour cette raison suspecte, on a dénié la raison et la parole, Elizabeth Costello revendique pour elle un discours qui ne reposerait justement pas sur des arguments philosophiques. La philosophie, on le sait, est du côté de l'homme, du côté de la raison et du langage. Dès lors, si l'on veut avoir une chance de comprendre ce que peut ressentir un animal, par exemple une chauvesouris, l'argumentation est de peu de secours ${ }^{23}$. Moins que l'imagination en tout cas. Or l'imagination n'est pas rien si on la prend au sérieux, c'est-à-dire si on ne la disqualifie pas d'emblée au bénéfice d'autres discours - par exemple philosophiques (ce qui est une première esquive) - et si l'on est capable d'assumer jusqu'au bout là où elle nous porte.

Le personnage d'Elizabeth Costello nous fait ainsi entrevoir de quelle autre façon nous pouvons nous esquiver devant la question animale nous les humains et nous les philosophes. La blessure que porte Costello est occasionnée par ce que Diamond appelle une «difficulté de la réalité»; en l'occurrence la difficulté soulevée par ce que nous faisons aux animaux tout en refusant de le voir et de l'imaginer ${ }^{24}$. C'est même par cette blessure du personnage que nous est rendue visible cette difficulté; rendue visible à travers les exemples littéraires qu'elle choisit plutôt que dans la

22 Le roman de Coetzee est une suite donnée aux Tanner Lectures à l'Université de Princeton en 1997 et paru en 1999 sous le titre The Lives of Animals. Pour une mise en perspective de ce texte, ainsi que de ceux qu'il a suscités, voir l'essai de Stephen Mulhall, The Wounded Animal, en particulier chap. 5: «Food for Thought», p. 69-94.

23 Sur le rôle et la place de l'argumentation en philosophie morale, je renvoie au texte que Cora Diamond a consacré à ce sujet: "Rien que des arguments?», in: L'Esprit réaliste, p. 391-415.

24 Cora Diamond appelle "esquive» ce que Derrida appelait pour sa part "méconnaissance» et "dénégation": Jacques Derrida, L’Animal que donc je suis, p. 46. Cf. aussi Florence Burgat, "La disparition», in: La Question animale, p. 135-145. 
discussion philosophique - ce dont ses interlocuteurs fictifs et réels ${ }^{25}$ ne veulent justement pas tenir compte, en assimilant trompeusement son discours à une argumentation en faveur de la libération animale. Il est vrai que la juxtaposition de ces deux interventions de Cora Diamond sur la question animale peut d'abord laisser une impression trompeuse. Elizabeth Costello n'est-elle pas en train d'effacer dans son discours les différences sur lesquelles la philosophe américaine voulait justement attirer notre attention? Ce serait manquer de voir plusieurs choses importantes: 1) d'une part, Elizabeth Costello ne parle pas au nom de la libération animale; 2) d'autre part, Elizabeth Costello en vient à mettre en question la pertinence même de l'argumentation philosophique. C'est bien depuis le corps qu'elle habite qu'elle parle et au nom de ce même concept "d'être vivant" que Diamond se proposait déjà de substituer à des considérations abstraites sur les intérêts et les droits des animaux.

Costello est une femme qui a conscience des limites de l'argumentation et c'est aussi une femme qui ose perdre ses concepts devant ce qui pour elle fait difficulté et qui est douloureux à penser. Si les animaux nous regardent sans pouvoir nous parler, l'argumentation restera toujours un procédé qui nous éloignera d'eux, un procédé pernicieux ${ }^{26}$. Pourtant il n'est pas dit qu'il faille nécessairement en passer par-là, par le langage et la raison (c'est-à-dire un langage argumenté, enchaîné selon l'ordre des raisons), pour accéder au point de vue de l'animal, pour accéder à ses souffrances et à ses désirs - ni même qu'il faille en passer par-là pour négocier avec eux le partage des territoires. Comme le rappelle Baptiste Morizot dans une discussion qui résonne étrangement avec le texte de Diamond, le diplomate est celui qui ose perdre ses concepts, qui doute de ses propres valeurs («incertain d'être humain», dit le texte de Coetzee) et qui s'aventure au-delà du langage de son pays d'origine,

25 Il est significatif en effet que les discussions philosophiques suscitées par le texte de Coetzee miment celles qui sont intégrées et répudiées par le roman. Sur ce point, voir les textes de philosophes recueillis dans The Lives of Animals, dont les réflexions (reflections) sont autant de déviations (deflections).

26 Voir le commentaire qu'en fait Ian Hacking dans le recueil de texte Philosophy and Animal life: «Diamond croit que philosopher sur les animaux détourne (deflects) la réalité d'une manière qu'elle juge pernicieuse» (p. 162, je traduis). 
en quête du non-sens de l'autre ${ }^{27}$. En parlant au nom des animaux, mais sans tomber dans les pièges de la discussion philosophique, Elizabeth Costello est, comme le singe savant de Kafka ${ }^{28}$ auquel elle s'identifie, une sorte de diplomate en terre étrangère, dans un pays en tout cas dont elle ne comprend plus l'ordinaire (l'ordinaire de notre indifférence par exemple) et où l'argumentation est insuffisante. C'est justement cela la difficulté: pour elle d'abord; pour nous, ensuite, qui essayons d'accéder à son point de vue $e^{29}$.

Je parle ici de diplomatie, encore que ce terme puisse être trompeur s'il nous induit à penser qu'Elizabeth Costello se fait l'avocate de la cause animale, reconduisant le genre de discussion qu'elle cherche à éviter. Si Costello est diplomate, c'est parce qu'elle doute que le langage (un certain usage du discours en tout cas) soit à la hauteur de la difficulté qu'elle entrevoit. Elle doit donc louvoyer avec les arguments et les concepts. Dès lors, c'est aux pouvoirs de l'imagination qu'elle se raccroche (et sur lesquelles elle compte) pour penser ce qui a pu être perdu (un concept de vivant qui existait et qui a disparu - que nous avons en tout cas rendu méconnaissable) et une réalité qui se refuse à être pensée (du moins avec des concepts rendus inutilisables, ne serait-ce que parce qu'il s'agit d'approcher un sujet qui n'en a pas toujours, ou pas les mêmes; autrement dit, parce que nous avons «un champ d'utilisation

27 Baptiste Morizot, Les Diplomates. Sur les rapports entre l'éthique et le non-sens de l'autre, je renvoie à l'éclairante présentation du texte de Cora Diamond par E. Halais et S. Laugier dans L'Importance d'être humain, ainsi qu'à l'article "Wittgenstein» du Dictionnaire d'éthique et de philosophie morale (sous la direction de Monique Canto-Sperber) rédigé par Cora Diamond.

28 Il est intéressant que dans la littérature et la théorie contemporaines, l'identification au singe Peter le Rouge indique souvent, de la part de celui ou celle qui reprend ce rôle, qu'il y a une difficulté de la réalité rendue manifeste, par-delà la dénégation de l'autre. Je pense en particulier au texte de Paul B. Preciado, Je suis un monstre qui vous parle, et à l'usage qu'il fait de la figure du singe parlant devant une société savante qui l'esquive, lui, ainsi que la difficulté de la réalité dont il est venu témoigner. Il va sans dire qu'il y a d'autres réalités que notre rapport à l'animal qui sont susceptibles de nous faire perdre nos concepts.

29 La question est alors la suivante: comment est-il possible pour nous d'accéder à son point de vue? Qu'est-ce que cela engage de notre part? 
étroit de leurs concepts $»^{30}$ ). Bien sûr, il n'est pas dit que l'on puisse recouvrer facilement des concepts perdus ${ }^{31}$ (des rapports à l'animal qui précéderaient les formes les plus graves de la domination exercée sur eux), ou que nous n'avons jamais eus, ni abandonner ceux qui déterminent la forme de vie dans laquelle nous sommes pris (une certaine confusion quant à nos manières de nous rapporter au vivant), mais il n'est pas dit non plus que ce soit impossible. Croire que c'est impossible, c'est justement se détourner du problème. Au fond, le scepticisme quant à la possibilité de se projeter ou de se transposer dans l'autre, le scepticisme quant aux possibilités de l'imagination est une forme de la mauvaise foi et de ce que, en général, Diamond appelle «esquive».

On comprend aussi pourquoi l'accusation d'anthropomorphisme dans le débat éthique et épistémologique sur l'animal a quelque chose d'égarant ${ }^{32}$. Tout se passe en effet comme si l'on ne savait trop quoi faire de l'anthropomorphisme, quoi faire d'une attitude qui serait bien trop généreuse avec l'animal, bien trop bête. Mais il se pourrait que ce soit une mauvaise façon de poser le problème ${ }^{33}$, une façon qui esquive

30 Cora Diamond, L'Importance d'être humain, p. 170.

31 J'emprunte cette expression de "concepts perdus" à l'article de Cora Diamond «Perdre ses concepts» reproduit dans L'Importance d'être humain. Un exemple de concept perdu est ce «monde conceptuel différent» dont témoignent les peintures de Paulus Potter dans lesquelles ne fait pas "difficulté» la proximité de l'homme avec l'animal ou du moins dans lesquelles cette «difficulté» n'est pas "esquivée» mais «exprimée» par l'idée de "maitrise" et d'autres concepts centraux du christianisme, voir p. 169. Mais si le monde conceptuel dans lequel de telles représentations prennent sens s'est éloigné, il peut néanmoins servir de "modèle» pour penser à nouveau frais notre relation à l'animal.

32 Cora Diamond, L'Importance d'être humain, p. 124.

33 Au demeurant, on ne songe pas assez au fait que l'anthropomorphisme, tel qu'il est parfois assumé par certains philosophes (au moins comme voie d'accès à l'animal, comme échelle qu'il faudrait ensuite rejeter) reste bien souvent centré sur l'homme; en particulier lorsqu'il est pensé comme l'une de ses prérogatives. Je pense à ce que dit Heidegger au $\$ 50$ des Concepts fondamentaux de la métaphysique: «Être transposé en d'autres fait partie de l'essence du Dasein humain" (p. 309). Comme si la question ne pouvait être posée de la projection inverse, du regard que l'animal porte sur nous et des biais que ce regard peut aussi contenir - quand cette capacité ne leur est pas, d'emblée, déniée. Le passage de l'animal visible à l'animal voyant (voyant non seulement son milieu, mais l'homme même sur lequel bien des 
justement "notre stupéfaction ${ }^{34}$ devant le fait que l'animal puisse nous être à la fois si semblable et si dissemblable. Car ce sont ces deux choses qu'il faut tenir - comme un diplomate doit parfois concilier des points de vue hétérogènes. La dénonciation coutumière de l'anthropomorphisme (lorsqu'il est répudié comme une attitude qu'il faut éviter, une bêtise donc) ne parviendra jamais à masquer toutes nos tentatives réussies pour nous placer imaginativement à la place de l'animal. Ceci d'autant moins que cette attitude est, en un sens, beaucoup plus naturelle ${ }^{35}$ et beaucoup plus réelle que son contraire. Mais elle ne l'est pas comme une «attitude naïve» ou un "préjugé» dont il faudrait ensuite se débarrasser - se débarrasser lorsque nous viendrait la raison ou la connaissance empirique (ou lorsqu'on passerait à table!). Car justement, c'est une attitude qui marche trop bien, trop bien avec les animaux. Au fond, c'est une attitude raisonnable. Comme le remarque la philosophe Mary Midgley: qu'il soit possible de savoir quel effet cela fait d'être un cheval ou un âne est une présupposition de l'acte même de domestiquer (de maîtriser ces animaux - ce qui signifie aussi maitriser quelque chose de leur vie conceptuelle ${ }^{36}$ ) - et par suite du pouvoir bien réel qui est exercé traditionnellement sur les animaux, sur certains d'entre eux du moins:

projections sont également possibles) est au cœur de la déconstruction de la question philosophique de l'animal dans le texte de Derrida.

34 J'utilise ici le terme de «stupéfaction» qui apparaît dans le texte de Cora Diamond, en rapport avec cette difficulté de la réalité. Cette stupeur, bien sûr, n'a rien à voir avec celle dans laquelle Heidegger croit pouvoir déterminer une essence de l'animal - cette stupeur qui est l'hébétude de l'abeille prise par la pulsion de butiner et qui signifie aussi la soustraction de toute forme de perception. La stupeur dont parle Cora Diamond est plutôt liée au vertige qui nous prend devant une réalité qui échappe aux concepts. Elle ne dit pas l'absence, l'inutilité ou l'impossibilité de la pensée - elle est en rapport avec une difficulté, ce qui est bien sûr tout autre chose.

35 Cette naturalité ne signifie pas que la projection anthropomorphique puisse se faire sans une observation patiente. Le modèle de cette observation patiente, qui va à l'encontre d'un anthropomorphisme spontané et trompeur, nous est offert par l'enquête éthologique réalisée par Thelma Rowell sur les moutons et sur leurs imperceptibles mouvements de museaux. Je renvoie ici aux analyses de Baptiste Morizot dans Les Diplomates, p. 164, qui en fait le modèle d'un anthropomorphisme méthodologique; et à l'article de Vinciane Despret, "Sheep Do Have Opinions», in: Bruno Latour, Peter Weibel (ed.), Making Things Public. Atmospheres of Democracy, p. 360-370.

36 Cora Diamond, L'Importance d'être humain, p. 170. 
«Les personnes qui réussissent à dresser les animaux ne le font pas du haut de cette supériorité abstraite et quelque peu magique qui est censément la leur, mais plutôt en entrant dans un rapport d'interaction sociale avec eux - en leur prêtant attention et en s'efforçant de comprendre comment les choses apparaissent du point de vue qui est le leur. Le fait d'ignorer ou de ne pas croire en l'existence de ce point de vue aurait pour effet de faire échouer leur tentative $»^{37}$.

Refuser la possibilité de nous projeter en l'animal nous conduirait sur une mauvaise piste dans la mesure où cela nous empêcherait de comprendre des phénomènes aussi massifs et aussi réels que l'élevage $e^{38}$ ou la domestication, que la chasse ou la pratique du pistage, que la constitution en général de ce qu'on nomme des communautés mixtes homme-animal ${ }^{39}$, une part importante de ce qui constitue, en fait, notre forme de vie.

À l'encontre du scepticisme, à l'encontre de l'idée alléguée par la philosophie selon laquelle il nous serait impossible d'accéder au point de vue de l'animal ${ }^{40}$, il y a donc la réalité des rapports sympathiques que nous entretenons au vivant - ces rapports mêmes qui sont

37 Mary Midgley, «La communauté mixte», in: Philosophie animale, p. 284.

38 Je distingue ici «l'élevage» des "productions animales» et de la zootechnie (dans ce qu'elle a justement de désanimalisant). Je renvoie aux travaux de Jocelyne Porcher, par exemple Etre bête (coécrit avec Vinciane Despret). Porcher montre que les éleveurs s'appuient sur des dispositions affectives sympathiques avec leurs animaux et des représentations de l'animal très proches de celle de l'être humain, en tant que vivants et habitants d'un corps.

39 En revanche, il n'est pas sûr que la manière dont on élève aujourd'hui les animaux conserve encore quelque chose de cette capacité de transposition, cet effort et cette réussite dans l'acte de se mettre «à la place de». Il va de soi aussi que ce qui se passe dans les fermes industrielles et les abattoirs ne correspond pas au concept de communauté mixte.

40 Je renvoie ici à l'article célèbre de Thomas Nagel, "Quel effet cela fait d'être une chauve-souris?», comme une illustration de cette attitude, intéressante dans la mesure où elle ne dénie pas à l'animal une subjectivité (tout au contraire). Il est symptomatique cependant que Nagel crée lui-même les conditions (philosophiques) à partir desquelles il n'est plus possible de répondre à la question qu'il pose. Ces conditions sont celles du mind-body problem. Mais alors, si l'animal n'est qu'un détour argumentatif, il s'agit bien encore d'une façon d'esquiver le problème. 
aujourd'hui si profondément bouleversés. En revanche, qu'un certain anthropomorphisme puisse être pertinent (y compris dans les sciences empiriques de l'animal $)^{41}$, que de multiples stratégies de projection soient chaque jour encore éprouvées (dans des négociations quotidiennes entre l'homme et l'animal) est compatible avec le fait que par ailleurs nous soyons capables de nous détourner tout à fait de l'animal, de sa souffrance ou de tout ce qui remplit l'idée d'être en vie ou d'avoir une vie. C'est que nous pouvons rejeter notre propre capacité à habiter un autre corps que le nôtre, quel que soit d'ailleurs ce corps: celui d'une chauve-souris, d'un animal d'abattoir, d'un détenu dans l'univers concentrationnaire ou d'un demandeur d'asile ${ }^{42}$. Et c'est cela qui est insupportable pour Elizabeth Costello, cela qu'elle éprouve comme une

41 Dans son essai consacré à la diplomatie lupine, Baptiste Morizot propose par exemple de distinguer entre deux versions de l'anthropomorphisme: l'une fautive, causée par le besoin de combler le vide et de remplir le silence (car l'animal restera, en un sens, toujours silencieux); l'autre heuristique et inventive, dont fait montre par exemple le travail de Rick McIntyre pour le Yellowstone Wolf Project et dont on aurait bien tort de se passer: cf. Baptiste Morizot, Les Diplomates, p. 162-163. Par ailleurs, il n'est pas interdit de procéder à l'inverse en apportant un éclaircissement sur nos relations affectives et sociales à partir de concepts empruntés à l'éthologie. Le concept de «dispersant" pourrait s'avérer très utile pour comprendre certains phénomènes économiques ou sociaux. Ainsi aussi de certains verbes comme "essaimer".

42 Certains contradicteurs, fictifs ou réels, d'Elizabeth Costello ont contesté son droit à parler de la Shoah - au motif que ce qui se passe dans les abattoirs ne pouvait pas réellement être comparé à ce qui s'est passé dans les camps de la mort - sans faire offense à la mémoire des juifs et déportés et sans faire un mésusage du concept de "ressemblance». Dans le roman de Coetzee, c'est la lettre envoyée par le poète Abraham Stern qui joue ce rôle. La suggestion de Cora Diamond est là encore éclairante. En vue de rendre visible une difficulté de la réalité, de l'exposer, affirme-t-elle, il arrive que l'on soit conduit à "faire le point" et diminuer la netteté de l'arrière-plan et ce faisant à passer à côté d'une autre difficulté - le genre précisément de difficultés que ne peuvent pas voir (pour d'autres raisons) les philosophes raisonnant abstraitement sur les intérêts et les droits des animaux. Cette nécessité de faire le point pour rejeter certaines difficultés à l'arrière-plan est un mécanisme qui épouse en fait une logique photographique. En revanche, cela ne prouve pas que s'intéresser à l'animal implique un désintéressement pour d'autres questions morales. Naviguer de la souffrance animale à la souffrance humaine ne revient pas à diminuer l'une ou l'autre souffrance. 
difficulté de la réalité: "Quelle sorte d'être sommes-nous pour y voir un problème?» demande-t-elle.

Il faut donc déplacer la question, d'une argumentation philosophique sur les droits et intérêts des animaux (Tom Regan et Peter Singer) ou d'une discussion philosophique sur les rapports entre corps et esprit, point de vue en première et en troisième personne ${ }^{43}$ (difficulté qui n'est d'ailleurs pas propre à, mais seulement aggravée par, la question animale), à une discussion sur nos pouvoirs de projection et d'imagination, sur la manière dont ces pouvoirs peuvent être inconsciemment oubliés ou rejetés. C'est ici que la littérature a toute sa place dans les discussions éthiques. Comme d'autres philosophes moraux de la seconde moitié du $\mathrm{xx}^{\mathrm{e}}$ siècle, Diamond adopte une démarche qui est à la fois éthique et esthétique, montrant qu'une partie au moins des difficultés morales auxquels nous nous confrontons mettent en jeu notre perception et notre capacité d'imagination sympathique; autrement dit, ne peuvent être résolues (lorsqu'elles le peuvent) qu’à partir du moment où nous faisons un effort pour voir autrement la situation, pour considérer le monde et ce qui s'y trouve avec une attention renouvelée ${ }^{44}$.

Par la littérature, suggère Diamond, (mais aussi par tout autre chemin que prendrait l'imagination - une imagination sollicitée par la nécessité pragmatique d'établir des relations avec les animaux dans des situations zoo-politiques, par le chamanisme ou par des pratiques qui nous sont plus familières telles que l'élevage ou la domesticité), nous pouvons ainsi nous rendre accessible notre propre sens de ce qu'est être un vivant et d'habiter dans un corps; un sens qui nous est disponible, quoi qu'en disent les philosophes attirés par le scepticisme ${ }^{45}$. D'où le

43 Je pense là encore au fameux article de Thomas Nagel, qui, sans traiter exactement du problème de l'anthropomorphisme ou de la question animale, a contribué à en fixer les termes dans le débat philosophique contemporain. D'où le fait que son nom soit mentionné par le personnage d'Elizabeth Costello comme symptôme d'une variété du scepticisme philosophique.

44 Pour une mise en perspective intéressante de ces questions dans le champ de la philosophie morale contemporaine, je renvoie à: Solange Chavel, Se mettre à la place d'autrui.

45 Il faut remarquer que l'éthologie ne partage pas le scepticisme des philosophes. Toute l'œuvre d'Uexküll peut ainsi être interprétée comme une tentative d'exploration des milieux animaux, une tentative de se donner leur point de vue (dans ce qu’il peut avoir de spécifique précisément, selon qu’on 
recours constant au poème, et dans le discours d'Elizabeth Costello (en particulier sa seconde intervention, qui justement ne prend pas la voie de la conférence philosophique), et dans les écrits de Cora Diamond. Que la littérature puisse se substituer au discours philosophique (ou le compléter) pour appréhender une "difficulté de la réalité " ${ }^{46}$ qui serait sinon esquivée devrait être un sujet de réflexion pour les philosophes. Comme je l'ai annoncé en introduction, il en va bien sûrdu rôle que peut jouer la philosophie: non seulement comme esquive (mais elle n'en a, bien sûr, nullement le monopole), mais aussi comme réflexion sur ces esquives. Au fond, et comme le dit d'emblée le titre du texte de Cora Diamond $^{47}$, la réalité, dans ce qu'elle peut avoir parfois d'impensable, est une difficulté aussi de la philosophie.

Difficulté de la philosophie, car elle peut contribuer à nous mettre à distance de nous-même et de nos propres capacités à éprouver ce que l'autre vit. À cet égard, il y a plus que des rapprochements fortuits entre les positions défendues par Cora Diamond et par Jacques Derrida ${ }^{48}$. Lorsque la philosophie ne veut pas aborder une difficulté, bien souvent elle retraduit ce qu'elle ne veut pas reconnaître comme un problème moral ou comme un problème épistémologique (les discussions formalistes en éthique animale d'un côté et certains arguments de philosophie de l'esprit de l'autre côté en offrent quelques illustrations). Ce que suggère Diamond, c'est de prendre le risque que notre pensée soit au contraire

s'intéresse au milieu de l'étoile de mer ou du choucas). Certes, apparaît ici et là dans les textes d'Uexküll une problématique de l'inconnaissable. Mais il convient de distinguer l'inconnaissable de l'inimaginable. Le rôle dévolu aux images dans le texte d'Uexküll démontre qu'il prend cela très au sérieux. De façon générale, il faut prendre garde à ce qu'une rhétorique du mystère et de l'impensable ne prenne le pas sur les façons très ordinaires que nous avons de nous représenter ce que vit le corps de l'autre (et pas seulement sa souffrance).

46 Ce n'est pas un hasard si cette expression utilisée par Cora Diamond est elle-même empruntée à l'écrivain américain John Updike.

47 Il s'agit du texte "Difficulté de la réalité, difficulté de la philosophie» intégré au recueil L'Importance d'être humain, mais qui était au départ la contribution de Cora Diamond à une série de conférences consacrée au texte de Coetzee.

48 C'est un point qui a été mis en avant par Cary Wolfe dans son introduction à Philosophy and Animal Life ainsi que dans l'article «Flesh and Finitude: Thinking Animals in (Post)Humanist Philosophy». 
déséquilibrée par la difficulté aperçue. Elizabeth Costello, parlant à partir de sa propre fragilité et souffrance, prend ce risque. Mais que signifie que notre pensée soit déséquilibrée, et en l'occurrence, qu'est-ce que cela signifie pour un philosophe? Il s'agit de faire l'expérience que certains de nos concepts, de nos manières ordinaires de parler et de désigner ne s'accrochent pas à la réalité, tournent à vide; autrement dit faire l'expérience (qui peut, en effet, être stupéfiante) que la réalité résiste à nos façons de classer les choses, de faire des partages, de nous représenter autrui ${ }^{49}$. Faire l'expérience aussi, à l'occasion d'une difficulté particulière, que notre façon habituelle de comprendre la vie conceptuelle est trompeuse, si elle consiste à imaginer que l'usage d'un concept est affaire seulement de découpage et d'étiquetage - des découpages et des étiquetages qui seraient déconnectés de la vie de ces concepts (leur histoire) et de notre vie avec les concepts (les actions, pensées et modes de compréhension qui s'adossent à eux). Faire l'expérience enfin que des concepts peuvent être abandonnés ou d'autres encore se perdre parce que la vie et le monde sur lesquels ils sont adossés ont disparu et, néanmoins, comprendre que ces concepts perdus, s'ils en valent la peine, peuvent être redécouverts et servir de modèle. Que toutes ces expériences ne puissent se réaliser sans le soutien de notre imagination n'indique pas qu'elles puissent se réaliser sans philosopher.

De ce point de vue, l'animal oppose bien une formidable résistance à notre pensée ${ }^{50}$ : non seulement les diverses formes de relation dans lesquelles nous sommes engagés avec lui et qui mettent au défi notre logique (des formes de relation si contradictoires qu'elles peuvent en effet avoir quelque chose de délirant), mais aussi l'infinie richesse de ses formes de vie. D'ailleurs, confronté à cette richesse, le premier concept qui s'effondre, c'est bien celui de l'animal - de «ce motanimal!» pour reprendre la formule de Derrida. Face à la perte de ce concept, à la perte

49 Et ce qui se produit alors est également une désintégration de notre tradition philosophique; désintégration manifestée dans ce zoo d'idées du discours prononcé par Elizabeth Costello. Sur ce point, $c f$. Lucie CAmpos, "Poétiques philosophiques de l'animal, W. G. Sebald et J. M. Coetzee», in: La Question animale, p. 259-273.

50 Que certaines difficultés de la réalité résistent à la pensée ne signifie pas que nous ne puissions pas les comprendre à partir de l'imagination. J'ai montré précédemment que notre capacité de nous projeter imaginativement dans les animaux était le plus souvent intacte. 
d'autres concepts encore (comme ceux de personne morale, de sujet de droit, de subjectivité), nous sommes renvoyés, affirme Diamond, à notre propre responsabilitée ${ }^{51}$ Cela peut occasionner une stupeur et une panique, comme à chaque fois qu'il en va seulement de notre responsabilité:

"nous sommes exposés - c'est-à-dire lancés dans la tâche de découvrir quelque chose avec quoi nous puissions vivre, et cela peut constituer au mieux une sorte de compromis amer ${ }^{52}$.

Au-delà de toutes les esquives auxquelles la philosophie peut participer (à côté d'autres discours), c'est donc aussi lorsque nous sommes arrachés à nos manières habituelles de penser qu'elle est surtout nécessaire. D'abord, en nous invitant à le reconnaître - nous invitant à reconnaître que nous avons des manières confuses de régler les difficultés que nous entrevoyons. Ensuite, en nous apprenant peut-être à mieux les penser: favorisant le développement d'une compréhension plus profonde des difficultés entrevues, nous invitant à faire des comparaisons et à en tirer certaines conclusions inattendues, attirant enfin notre attention sur certains aspects de la réalités3. Aucune voie n'est plus recommandable qu'une autre (la déconstruction derridienne, le perspectivisme, l'approche diplomatique, l'horizon wittgensteinien dans lequel s'engage, pour sa part, Diamond), du moment que la philosophie a d'abord pris la mesure de ce qui lui reste encore à penser.

51 Cette idée de responsabilité est empruntée à la philosophie de Cavell et à l'idée que face à des critères qui peuvent s'avérer décevants ou que l'on peut tout à coup suspendre, c'est à nous que revient la responsabilité de l'usage que l'on fait des mots et des concepts: par exemple des mots et des concepts "avoir un corps, avoir une vie, souffrir, etc.». Sur ce point je renvoie à la première partie des Voix de la raison. Comme l'affirme Stanley Cavell «moi seul pourtant puis atteindre à cet intimité, en l'acceptant d'abord pour hôte de mes concepts de l'âme humaine", in: Stanley Cavell Les Voix de la raison, "Wittgenstein et le concept de connaissance humaine», p. 141.

52 Cora Diamond, L'Importance d'être humain, p. 301.

53 Voilà quelques aperçus de la façon dont Cora Diamond conçoit le rôle de la philosophie, en tout cas de l'activité de pensée. Voir en particulier L'Esprit réaliste, p. 409-410. 


\section{Bibliographie}

BaIlly Jean-Christophe, Le Parti pris des animaux, Paris: Christian Bourgois, 2013.

Bimbenet Etienne, Le Complexe des trois singes. Essai sur sur l'animalité humaine, Paris: Le Seuil, 2017.

Burgat Florence, «La disparition", in: Chapouthier Georges, Coquio Catherine, Campos Lucie, Engélibert Georges (dir.), La Question animale, Rennes: PUR, 2011, p. 135-150.

Campos Lucie, «Poétiques philosophiques de l'animal, W. G Sebald et J. M. Coetzee", in: Chapouthier Georges, Coquio Catherine, Campos Lucie, EngÉlibert Georges (dir.), La Question animale, Rennes: PUR, 2011, p. 259-274.

Cavell Stanley, Diamond Cora, Mcdowell John, Hacking Ian, Wolf Cary, Philosophy and Animal Life, New-York: Columbia University Press, 2008.

Cavell Stanley, Les Voix de la raison, trad. S. Laugier et N. Balson, Paris: Seuil, 1996.

Chavel Solange, Se mettre à la place d'autrui, Rennes: PUR, 2012.

Coetzee John Maxwell, The Lives of Animals, Princeton: Princeton University Press, 1999 [conférence partiellement reprise dans Elisabeth Costello, tr. C. Lauga du Plessis, Paris: Le Seuil, 2004].

Derrida Jacques, L'Animal que donc je suis, Paris: Galilée, 2006.

Despret Vinciane, Porcher Jocelyn, Etre bête, Arles: Actes Sud, 2007.

Diamond Cora, L'Esprit réaliste: Wittgenstein, la philosophie de l'esprit, trad. E. Halais et J.-Y. Mondon, Paris: PUF, 2004.

Diamond Cora, L'Importance d'être humain et autres essais de philosophie morale, trad. E. Halais, J.-Y. Mondon, et S. Laugier, Paris: PUF, 2011.

Diamond Cora, article "Wittgenstein" du Dictionnaire d'éthique et de philosophie morale, sous la direction de M. Canto-Sperber, Paris: PUF, 1996, p. 1626-1633.

Francione Gary, "Prendre la sensibilité au sérieux", in: Afeissa Hicham-Stéphane, Jeangene Vilmer Jean-Baptiste (dir.), Philosophie animale: différence, responsabilité et communauté, Paris: Vrin, 2010, p. 185-223.

Heidegger Martin, Concepts fondamentaux de la métaphysique [1929], traduction D. Panis, Paris: Gallimard, 1992.

IsaAcs Susan, Intellectual Growth in Young Children, London: Routledge, 1930.

Midgley Mary, "La communauté mixte», in: Afeissa HichamStéphane, Jeangene Vilmer Jean-Baptiste (dir.), Philosophie 
animale: différence, responsabilité et communauté, Paris: Vrin, 2010, p. 281-309.

Morizot Baptiste, Les Diplomates, Marseille: Wildproject, 2016.

Mulhall Stephen, The Wounded Animal, Princeton: Princeton University Press, 2009.

Nagel Thomas, Questions mortelles, trad. P. Engel et C. Tiercelin, Paris PUF, 1983.

Regan Tom, "Pour les droits des animaux", in: Afeissa HichamStéphane, Jeangene Vilmer Jean-Baptiste (dir.), Philosophie animale: différence, responsabilité et communauté, Paris: Vrin, 2010, p. 161-185.

Ross William David, The Right and the Good, Oxford: The Clarendon Press, 2002.

Singer Peter, "Libération animale ou droit des animaux?», in: Afeissa Hicham-Stéphane, Jeangene Vilmer Jean-Baptiste (dir.), Philosophie animale: différence, responsabilité et communauté, Paris: Vrin, 2010, p. 137-161.

Von Uexкüll Jakob, Milieu animal et milieu humain, Paris: Rivage, 2010.

Wolfe Cary, «Flesh and Finitude: Thinking Animals in (Post)humanist Philosophy", SubStance, vol. 37, n 3, p. 8-36. 